\title{
Estimating capacity and resource allocation in healthcare settings using business process modelling and simulation ${ }^{1}$
}

\author{
G. A. Redeker ${ }^{1,3}$, T. Webber ${ }^{1}$, R. M. Czekster ${ }^{1}$, S. Quickert ${ }^{2}$, J. K. F. Bowles ${ }^{2}$ \\ ${ }^{1}$ University of Santa Cruz do Sul (UNISC), Santa Cruz do Sul, RS - Brazil \\ ${ }^{2}$ School of Computer Science, University of St Andrews, St Andrews - UK \\ ${ }^{3}$ Hospital Bruno Born (HBB), Lajeado, RS - Brazil \\ guilhermeahbb.com.br, \{thaiscs,ricardoc\}@unisc.br, \{sq21,jkfb\}@st-andrews.ac.uk
}

\begin{abstract}
Resumo. Serviços de saúde envolvem tomada de decisões complexas desde o planejamento até o gerenciamento de recursos. Os hospitais alocam recursos conforme a experiência dos gestores, entretanto, devido a complexidade dos processos envolvidos, as decisões contém muitas incertezas, variabilidades e restrições. Sistemas de informações devem ser robustos para prover suporte a gerência e serem capazes de controlar e suportar processos de trabalho. O presente trabalho explora a sinergia necessária combinando gestão de processos e simulação. O objetivo é estimar índices de desempenho considerando o gerenciamento da capacidade de um centro cirúrgico como estudo de caso.
\end{abstract}

\begin{abstract}
Healthcare involves complex decision making from planning to resource management. Resources in hospitals are usually allocated by experienced managers, however, due to an inherent process complexity, decisions are surrounded by uncertainties, variabilities, and constraints. Information Systems must be robust enough to provide support to stakeholders, capable of controlling and support work flows. The present work explores the required synergy when combining business processes with discrete event simulation. The objective is to estimate performance indices and address capacity management of a surgical center as a case study.
\end{abstract}

\section{Introduction}

Healthcare management is currently facing substantial pressure for maintaining highly efficient and optimised processes throughout hospitals and healthcare providers. Understanding and coping with such complex activities must be dealt with through user-friendly information systems that are transparent to end operators in large healthcare settings. In a typical hospital care pathway, several patients with distinct conditions and illnesses must undergo various consultations with medical doctors (among other tasks), which in turn must abide to protocols and processes. It is challenging to map processes into realistic models using modelling notations combined with reliable operational data.

One standard formalism to enlarge the knowledge and documentation regarding processes is Business Process Modelling Notation (BPMN). The notation was developed to be applicable to a variety of domains, offering very simple but a wide range of visual notation to capture process activities (Dumas et al. 2013; Geiger, Wirtz, and Weberei 2013). Another extensively used technique to promote reasonable use of resources from models for performance assessment is Discrete Event Simulation (DES). Analysts can build sets of 'what if' scenarios that compute alternative outcomes for studying better process configurations. This is advantageous, since managers (and other roles) could play with processes, tasks, resources and service times, calculating performance indices and Key Performance Indicators (KPIs) (Bisogno et al. 2016, Khalifa and Khalid 2015, Rossetti 2009).

The main contribution of our paper is to discuss how to combine high level models written in

\footnotetext{
${ }^{1}$ Supported by the RAE under the Newton Research Collaboration Programme (NRCP1617/5/62).
} 
BPMN with DES, in such a manner that managers could profit from estimated performance scenarios as decision support data. Our approach can help predict sets of behaviours through the analysis of multiple scenarios looking at historical data collected from the ERP (Enterprise Resource Planning) system mixed with indirect observation of processes and semi-structured interviews.

We chose a surgical centre of a medium sized hospital located at the southern region of Brazil as our case study. We aim to show the steps involved in (i) acquiring knowledge about the process itself using BPMN, and (ii) translate the high-level BPMN model to an executable simulation model, parameterized with actual ERP system data plus observations. We also discuss the potential benefits of devising a straightforward decision support tool based on BPMN and DES focusing on userfriendly interfaces and real-time responses to configuration scenarios.

\section{Surgery case study: blending healthcare business process model with DES}

Surgery practices are key operations associated with high costs and risks. It is very important to thoroughly study such processes, since they relate to patients' lives and draw attention from management due to its financial concerns. Surgery incorporates different medical-related staff (e.g. doctors, nurses, cleaning staff), and procedures (for instance, appendectomy, pericardiectomy). Such processes are very rich for analysis, with direct impact on business outcomes, use of resources analysis, and patient satisfaction. For example, the required time to perform a surgery depends on the patient profile (age group, health preconditions), the type of procedure, and necessary equipment and supplies. It is very difficult to incorporate all these aspects into a simulation model whilst keeping the model realistic. However, it is still possible to focus only on the surgery process, analysing key scenarios. For example, for surgery, it is common to inspect KPIs such as Bedroom (i.e. Operating Theatre) Occupancy Percentage, Average Time, Average Delay Time, Time Wasted Preparing Room, etc.

The BPMN mapping comprises the study of each activity with its actors (e.g. roles) as well as locations (surgery room) and allocated resources. Figure 1 depicts the BPMN model from Camunda Software Suite ${ }^{2}$. According to Figure 1, the process begins with the patient's' arrival to the surgical centre, where she is prepared for surgery by nurses (e.g. installing devices for monitoring and administer pre-surgical medications). The patient then wait for an operating theatre to be ready. Depending on the procedure, other professionals may be required such as anesthetists and operating department practitioners. When both the operating theatre and the team are ready, the patient is operated. After surgery, a patient is discharged if the health is consistent and if no problem has occurred during or post surgery. At the same time as patients are recovering, cleaning professionals prepare the operating theatre for the next patient. The cleaning professionals are very important because they prepare the operating theatre for the next patient, and the best case scenario is when they readily start working right after the surgery has taken place. This, however, is very difficult to attain, because they work across different hospital sectors and perform other duties. It is also rather obvious that medical doctors and their teams play a key role in performance, since if a single one is missing for a procedure, the patient's operation would have to be postponed. It is very important to keep the rooms busy at all times and maintain the delays to low levels, because it impacts directly on patient satisfaction and welfare, as well as process efficiency.

\footnotetext{
${ }^{2}$ Camunda is an open and free software available for download at https://camunda.com/
} 


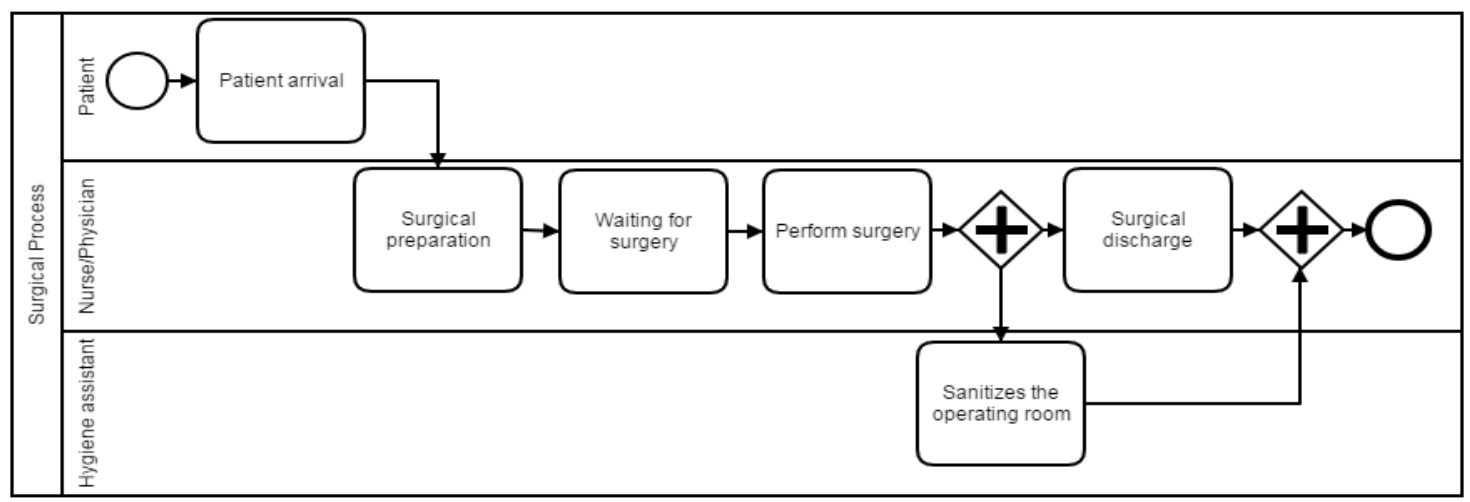

Figure 1. BPMN model for surgery in a hospital.

The BPMN model is manually translated to a compatible discrete-event simulation model. For illustration purposes, we have used Arena Software Suite ${ }^{3}$ to provide a simulation based on BPMN model and annotations. Figure 2 below presents our model of Surgical Rooms with actual interarrival times for patients extracted from ERP system. Activities in the BPMN model correspond to processes in the simulation, and start/end events are converted to specific modules responsible for the creation and disposal of entities during execution. The simulation model is thus consistent keeping the same flow of activities found in the BPMN model. It is important to have a validated dataset to obtain the required parameters for the simulation. The following data was specifically retrieved and processed (after removing invalid entries, missing data and null fields, and so on) and consists of a two year long longitudinal study, list of assigned resources, and procedure identifier. Datetime format is processed to be accepted by the probability distribution fitting tool inside Arena Software called Input Analyzer (this tool is shipped with the Arena main software bundle).

The simulation model has produced an initial model, called AS-IS model, which maps the actual operation, i.e., a snapshot of the current status of the situation under study. We have compared it against the data to see whether the performance indices were a close match. The AS-IS scenario showed that we have achieved similar results in terms of throughput and waiting times for the modelled queues, validating our base model. Examples of parameters such as average time between arrivals for activities are: Surgical preparation takes an interval of 4.81 to 6.93 minutes; Sanitization of the operating room is set at a fixed on 15 minutes (simulators do allow some stochastic fluctuation, e.g. using probability distributions); Waiting time for surgery around 14 to 33 minutes; and surgeries around 38.83 to 102.66 minutes (depending on complexity could last more minutes or hours to be accomplished). Analyzing rooms' occupancy, the simulation provided total times from 95.38 to 449.83 minutes depending on the room. The worst waiting time for surgery was computed as 180.83 minutes for a given patient considering around 12.13 minutes for a specific room setup. For this simulation we used a dataset containing around 9500 surgeries, without differentiating their scheduled time during the day. The resources considered hygienic assistants (assigned to room setup activity), a physician, an anesthesiologist and a surgical technician. All resources were involved in the surgical procedure. In the room setup, the hygienic assistant was busy on average $60.7 \%$ of the time considering 6 rooms. For surgery we have looked the use of the rooms and we computed those measures to be between $4.3 \%$ and $82.5 \%$ of the time. Such variation is a good indication of where to focus efforts to keep reasonable levels of operating rooms as patients' demands are varying.

\footnotetext{
${ }^{3}$ Arena Software Suite is proprietary software, needing a license to work. Website: https://www.arenasimulation.com/
} 


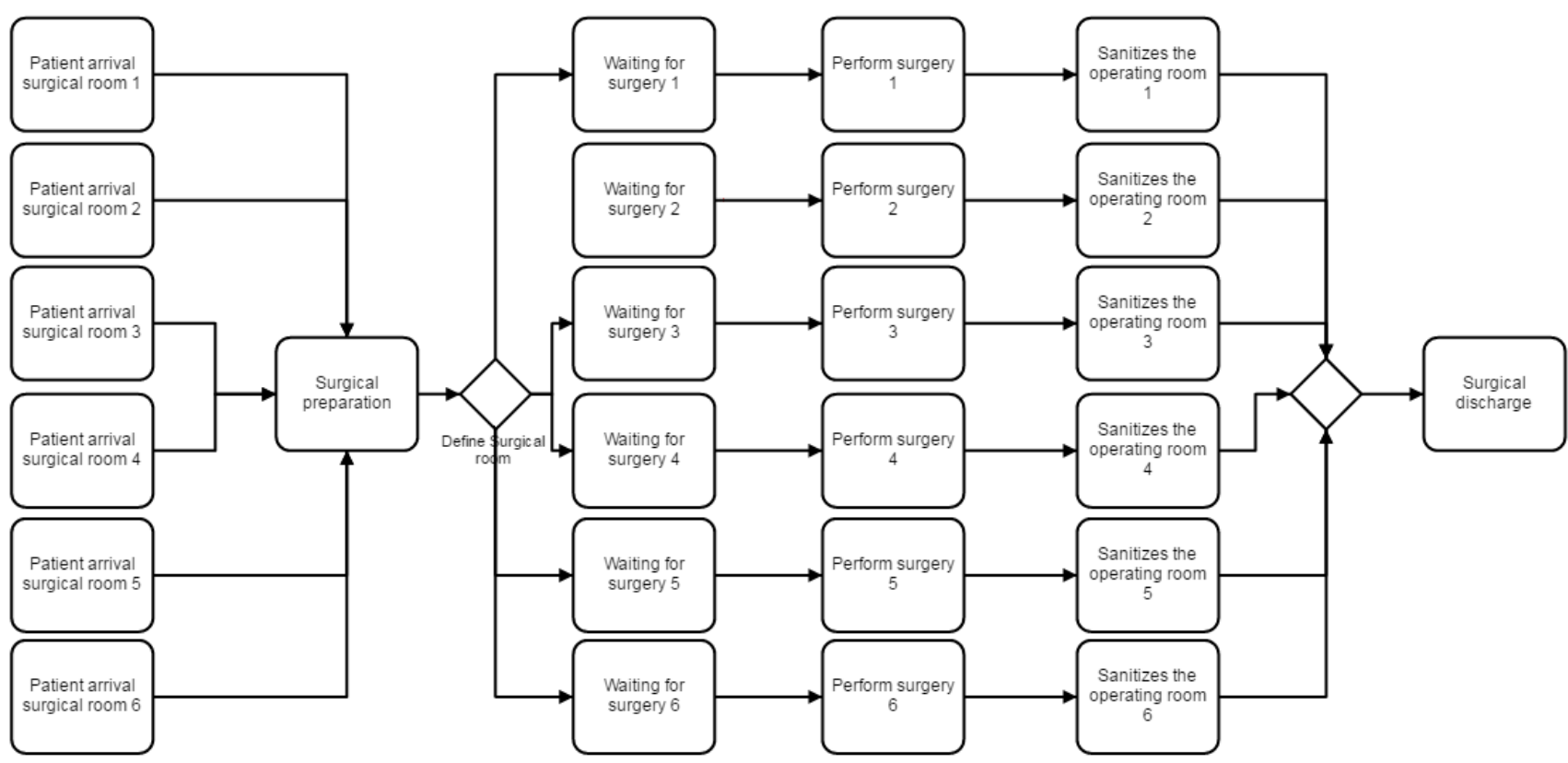

Figure 2. Arena-based model schema: BPMN mapping to DES model

\section{Final considerations}

The area of quantitative assessment of systems is a crucial technique to help stakeholders make quick and reasonable decisions given available information. With BPMN and DES integrated in a decision support tool, managers can choose better throughputs or lesser waiting times. They could rearrange resources as well as professionals involved at different stages of a process to ensure high levels of productivity. With simulation, this view is broadened and it is possible not only to map and address such deficiencies but to reassess entire operations to meet performance expectations. This work in progress has already used modelling and simulation to map surgery procedures to a high level model in BPMN. We devise a compatible simulation model to aid the exploration of configurations yielding performance results in terms of resource utilisation and waiting times. Managers should increase their awareness towards simulation practices as a valid alternative for system analysis. As future work, we will develop an automatic model generation and scripting tool to analyse scenarios outputs, presenting stakeholders with more information to make better decisions.

\section{References}

Bisogno S. et al. (2016). Combining modelling and simulation approaches: How to measure performance of business processes. Business Process Management Journal, vol. 22(1), pp 56-74.

Dumas, M. et al. (2013). Fundamentals of business process management (Vol. 1 \& 2). Springer.

Geiger M., Wirtz G. and der Weberei A. (2013). BPMN 2.0 Serialization-Standard Compliance Issues and Evaluation of Modeling Tools. In: EMISA 2013, pp 177-190.

Khalifa, M. and Khalid, P. (2015). Developing Strategic Health Care Key Performance Indicators: A Case Study on a Tertiary Care Hospital. Procedia Computer Science, vol. 63, pp 459-466.

Rossetti, M. D. Simulation Modeling and Arena. (2009). Wiley Press, 2nd Edition. 